\title{
Asymmetric Hovering Flapping Flight: a Computational Study
}

\author{
Jardin Thierry, Farcy Alain and David Laurent \\ LEA, University of Poitiers, CNRS, ENSMA
}

France

\section{Introduction}

In the early 90's, Micro Air Vehicles (MAV's) appeared as a possible solution for missions of reconnaissance in constrained environments. The American Defense Advanced Research Projects Agency (DARPA) initiated workshops on the concept and, in 1997, raised funds to conduct a multi-year programme whose objective was to develop a low cost, high autonomy unmanned aircraft, with a largest dimension limited to 15 centimeters (6 inches). In terms of aerodynamics, this typical size specification places the corresponding airflow in the range of low Reynolds number flows, typically between $10^{2}$ and $10^{4}$. Several prototypes were tested, based on the conventional fixed and rotary wing concepts. However, at such low Reynolds numbers and notably supported by the researches carried on the analysis of insects' flight, the flapping wing concept appeared as an alternative answer, suggesting enhanced aerodynamic performances (lift, efficiency), flight agility, capability to hover coupled with a low noise generation. The latter arises from the complex wing motion defined by superimposed translating (downstroke and upstroke) and rotating (supination and pronation) motions.

Pioneer works relying on the aerodynamics of flapping wings were proposed by biologists whose objective was to evaluate the amount of lift generated by insects. After several attempts based on the quasi-steady approach, it was admitted that unsteady aerodynamic mechanisms are essential to keep an insect aloft, especially while hovering (Jensen, 1956; Weis-Fogh, 1973; Ellington 1984). Precisely, three phenomena may be distinguished:

1. The presence of a leading edge vortex (LEV or dynamic stall mechanism) during the translating phases, acting as a low pressure suction region on the extrados of the wing. Due to its importance in aeronautics, this phenomenon was extensively studied experimentally (Walker, 1931) and analytically (Polhamus, 1971) before its evidence was demonstrated and analysed in the context of flapping wings (Maxworthy, 1979; Dickinson \& Götz, 1993).

2. The Kramer effect, assimilated to the supplementary air circulation resulting from the combined translating and rotating motions (Kramer, 1932; Bennett, 1970; Dickinson et al, 1999).

3. The wake capture mechanism, occurring as the wing encounters and interacts with its own wake generated during previous phases (Dickinson, 1994; Dickinson et al, 1999).

One should keep in mind that the spatial and temporal behaviours of such unsteady phenomena highly depend on the wing kinematics. Thus, when analysing the flow 
dynamics generated by a flapping wing, it is essential to clearly precise the flight configuration studied. Basically, two main approaches may be distinguished. The first one is the forward-flapping flight configuration for which the wing flaps into a head wind. The resulting aerodynamic force can be divided into two components: the forward (horizontal) and the upward (vertical) components which must be sufficiently strong to counter the body drag force and the weight respectively. The second one, referred to as the hovering-flapping flight configuration, is in fact an extreme mode of flight where the head wind velocity is zero. The resulting mean aerodynamic force is here strictly vertical, the average horizontal force over a flapping period being null. In this particular case, the unsteady effects are preponderant relatively to the quasi steady effects. Considering hovering flapping flight leads to further differentiation whether the wing flaps along a horizontal stroke plane (symmetric or normal hovering) or an inclined stroke plane (asymmetric hovering). The former case has been under much consideration since it appeared to be the most common configuration observed in the world of insects. Specifically, the influence of various kinematic parameters (e.g. angle of attack, position of the centre of rotation, Reynolds number etc) was experimentally (Sane \& Dickinson, 2001; Sane \& Dickinson, 2002; Kurtulus, 2005) and numerically (Wu \& Sun, 2004; Kurtulus, 2005) analysed, giving satisfying agreement. On the other hand, asymmetric hovering studies seemed restricted to biologic configurations (reproducing the wing kinematics of the dragonfly) and very few works reported parametrical results (Wang, 2004) dedicated to MAVs improvement. Yet, introducing asymmetry in hovering flapping flight is an appealing approach as the lifting force results from the combination of both lift and drag, presupposing enhanced aerodynamic efficiency.

In this chapter, two dimensional numerical computations are used to evaluate the flow dynamics and the resulting aerodynamic loads experienced by a wing undergoing asymmetric hovering flapping motions at Reynolds 1000. On the contrary to previous works, the present parametrical analysis focuses on the aerodynamic performances of nonbiological configurations for application to Micro Air Vehicles. Reference to a larger context is ensured by comparing asymmetric configurations with the widely studied symmetric configurations.

\section{Flapping kinematics and parameters}

Normal (or symmetric) flapping motions are characterized by strictly opposed downstroke and upstroke wing kinematics. As a consequence, the drag (aerodynamic force component collinear to the stroke plane) generated during downstroke counteracts the drag generated during upstroke. If the stroke plane is set as the horizontal, the drag may be assimilated to the horizontal force component whose mean value over a period is hence null, ensuring the hovering condition. In this study, asymmetry is introduced by fixing different downstroke and upstroke angles of attack. The resulting asymmetric motions exhibit dissimilar downstroke and upstroke drag such that setting the stroke plane as the horizontal no longer ensures the hovering condition. Thus, the latter should be tilted (angle $\beta$ ), resulting in a combined action of both lift and drag as the effective lifting (or vertical) force.

A set of symmetric and asymmetric cases is analysed for a two dimensional NACA0012 profile. The wing kinematics result from the combination of translating and rotating motions as shown in figure 1. Basically, the flapping motions may be decomposed into different regions whether the wing is translating at constant speed and fixed angle of attack 
(region $\mathrm{T}$ ) or is submitted to both varying translation speed and rotating motion (regions $\mathrm{R}$ ). Region $\mathrm{T}$ and regions $\mathrm{R}$ are respectively 4 and 1 chord long, so that the wing travels along a total course of 6 chords during one stroke. The rotation is applied around a spanwise axis located $1 / 4$ chord away from the leading edge. The constant wing velocity $V_{0}$ reached during the pure translation phases (region T) is calculated with respect to the Reynolds number such that:

$$
\operatorname{Re}=\frac{\mathrm{cV}_{0}}{\mathrm{v}}
$$

where $\mathrm{c}$ is the chord of the NACA0012 profile and $v$ the kinematic viscosity of air. Note that the Reynolds number represents the adimensional ratio between inertial and viscous forces and is here fixed to 1000 such that the flow is considered to be laminar. The flapping period $\mathrm{T}$ is defined as:

$$
\mathrm{T}=\frac{4 \mathrm{c}}{\left|\mathrm{V}_{0}\right|}\left(2+\frac{\Pi}{2}\right)
$$

leading to a flapping frequency of approximately $10 \mathrm{~Hz}$. The varying parameters are chosen to be the downstroke and upstroke angles of attack $\left(\alpha_{d}, \alpha_{u}\right)$, the difference between the two reflecting the asymmetry of the motion. In order to ensure the continuity of the translating and rotating accelerations as necessary to numerically solve the Navier-Stokes equations, the instantaneous velocities follow $4^{\text {th }}$ order polynomial motion laws as displayed in figure 1.
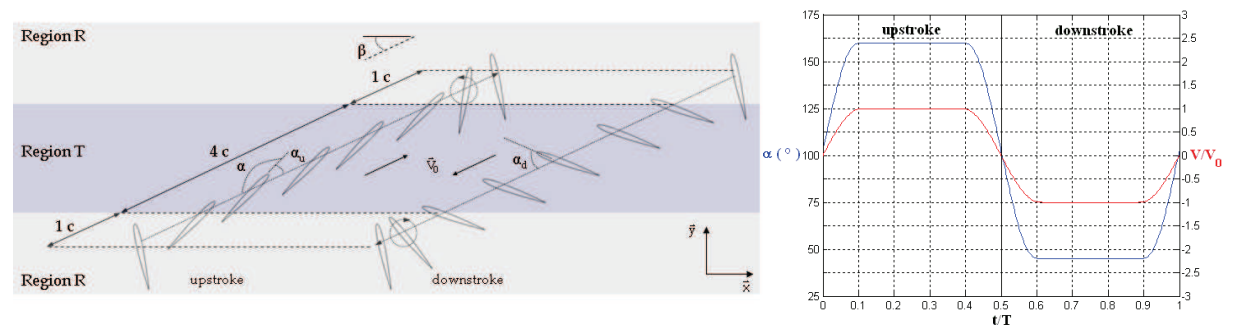

Figure 1. Schematization of the flapping kinematics and time evolution of the angle of attack and translating velocity $\left(\alpha_{\mathrm{d}}=45^{\circ}, \alpha_{\mathrm{u}}=20^{\circ}\right)$

\section{Investigation tools}

\subsection{Numerical solver}

The aerodynamic flow established in the vicinity of the airfoil is computed using a finite element method. The two dimensional time-dependent Navier-Stokes equations (equations (3) and (4) in Cartesian tensor notation for general compressible and incompressible flows) are directly solved (DNS) in the fixed inertial reference frame through a moving mesh method, assuming laminar incompressible flow.

$$
\frac{\phi}{\partial}+\frac{\partial}{\partial x_{j}}\left(\rho u_{j}\right)=0
$$




$$
\frac{\partial p u_{i}}{\partial t}+\frac{\partial}{\partial z_{j}}\left(\rho u_{j} u_{i}-\tau_{i j}\right)=-\frac{\partial}{\partial z_{i}}
$$

with the constitutive relation for a laminar Newtonian fluid:

$$
\begin{gathered}
\tau_{\mathrm{ij}}=2 \mu \mathrm{s}_{\mathrm{ij}}-\frac{2}{3} \mu \frac{\partial \mathrm{u}_{\mathrm{k}}}{\partial \mathrm{k}_{\mathrm{k}}} \delta_{\mathrm{ij}} \\
\mathrm{s}_{\mathrm{ij}}=\frac{1}{2}\left(\frac{\partial \mathrm{u}_{\mathrm{i}}}{\partial \mathrm{k}_{\mathrm{j}}}+\frac{\partial \mathrm{at}_{\mathrm{j}}}{\partial \mathrm{k}_{\mathrm{i}}}\right)
\end{gathered}
$$

Symbols are listed in table 1 below.

\begin{tabular}{|l|l|}
\hline$t$ & time \\
\hline$x_{i}$ & Cartesian coordinate \\
\hline$u_{i}$ & Fluid velocity component \\
\hline$p$ & Piezometric pressure \\
\hline$\rho$ & density \\
\hline$\mu$ & Molecular dynamic viscosity \\
\hline$\tau_{i j}$ & Stress tensor components \\
\hline$s_{i j}$ & Rate of strain tensor \\
\hline$\delta_{i j}$ & Kronecker delta \\
\hline
\end{tabular}

Table 1. Definition of the Navier-Stokes equations symbols

The Navier-Stokes equations are solved on a non-conformal O-type grid as shown in figure 2. The latter is composed of 57500 cells, forming a refined rectangular domain close to the profile and a coarser circular domain at the far field. The instantaneous airfoil velocity is computed by means of user-defined subroutines and implemented through a no-slip boundary condition. The pressure is fixed and assumed to be the standard air pressure at the far field, located 15 chords away from the airfoil centre of rotation. The velocities at the corresponding cell faces are linked to the local pressure gradients, allowing the local flow to be inwards or outwards. For a two-dimensional configuration, symmetric boundary conditions are applied on the front and back side of the grid. The spatial and temporal discretisations are performed using an upwind differencing scheme and a PISO scheme respectively. The PISO approach consists of a special implicit scheme, based on the fully implicit Euler scheme and explicit deferred correctors, which results in a formal accuracy lying between first and second order. Setting 1000 time steps per flapping period leads to a maximum and mean Courant number (CFL) of approximately 10 and 0.5 respectively. Note that the results exposed in the present paper concern the 7th flapping period for which the flow is ensured to be periodical.

Higher resolution and larger computational domain tests (keeping a similar CFL) demonstrated that the solution is mesh independent. 

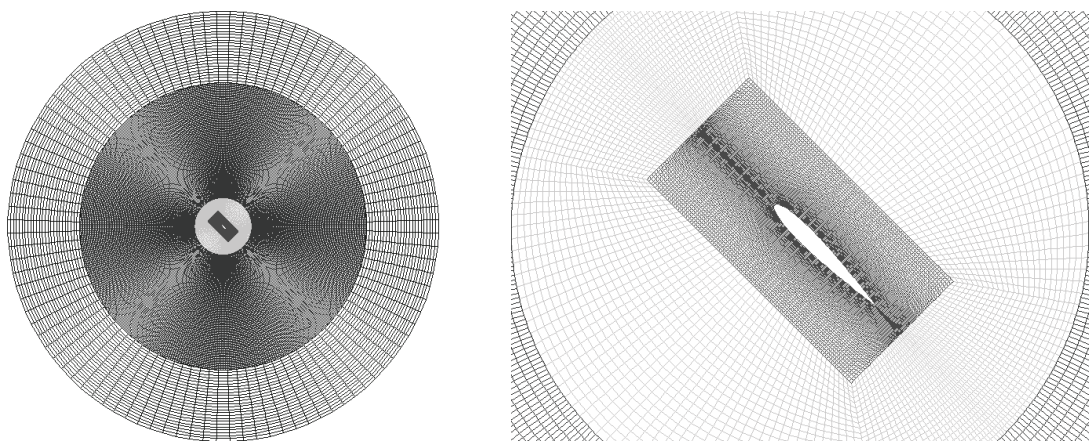

Figure 2. General and zoomed view of the computational grid

\subsection{Vortex characterization}

The numerical flow solver returns the velocity and the pressure flow fields in the whole computational domain at each time step. In order to characterize the flow dynamics, it is convenient to derive specific criteria which facilitate the vortex cores identification. The present analysis focuses on the $\lambda_{2}$ criteria (Jeong \& Hussain, 1995), whose definition for a two-dimensional flow in the $x y$ plane of velocity components uv is:

$$
\lambda_{2}=\frac{\partial \mathrm{u}}{\partial \mathrm{y}} \frac{\partial}{\partial x}-\frac{\partial \mathrm{u}}{\partial \mathrm{x}} \frac{\partial}{\partial \mathrm{y}}
$$

On the contrary to the vorticity, the $\lambda_{2}$ criterion differentiates the strain regions from the vortex core regions. Precisely, $\lambda_{2}$ is the second highest eigenvalue of the tensor $\Omega^{2}+S^{2}$, where $S$ and $\Omega$ are respectively the symmetric and antisymmetric components of the velocity gradient tensor. Negative values of $\lambda_{2}$ put into evidence local minima of pressure usually illustrating vortex cores.

\subsection{Aerodynamic loads}

The presence of vortical structures in the vicinity of the flapping airfoil highly influences the generation of aerodynamic loads. As will be exposed in the following section, vortex cores are usually assimilated to low pressure regions which may act as lift enhancers if located on the extrados of the airfoil. As a consequence, the understanding of flapping airfoil performances imposes to establish a correlation between the vortex behaviours and the resulting aerodynamic force $\mathrm{F}$. The latter is inferred by integrating both pressure and viscous stresses along the wing surface and may be decomposed into the lift $F_{1}$ and drag $F_{d}$ components (respectively orthogonal and collinear to the stroke plane) or the vertical $\mathrm{F}_{\mathrm{y}}$ and horizontal $\mathrm{F}_{\mathrm{x}}$ components, linked by the following relations:

$$
\begin{gathered}
F_{x}= \pm F_{d} \cos \beta-F_{1} \sin \beta \\
F_{y}=F_{1} \cos \beta \pm F_{d} \sin \beta
\end{gathered}
$$

The basic two-dimensional aerodynamic coefficients are obtained by adimensionalizing the previous components using the constant translating velocity $\mathrm{V}_{0}$ and the chord of the airfoil c: 


$$
\mathrm{C}_{\mathrm{i}}=\frac{2 \mathrm{~F}_{\mathrm{i}}}{\rho \mathrm{\rho c} \mathrm{V}_{0}^{2}}
$$

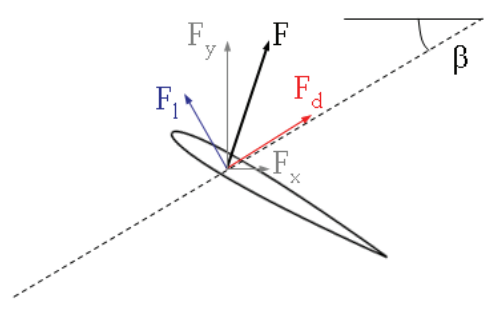

Figure 3. Relation between the resulting aerodynamic force $\mathrm{F}$ and the various components $\mathrm{F}_{\mathrm{l}}, \mathrm{F}_{\mathrm{d}}, \mathrm{F}_{\mathrm{y}}$ and $\mathrm{F}_{\mathrm{x}}$

As a first approach, it is of interest to evaluate the mean values of these coefficients by averaging them over a flapping period $\mathrm{T}$ :

$$
\overline{\mathrm{C}_{\mathrm{i}}}=\frac{1}{\mathrm{~T}}{ }_{0}^{\mathrm{T}} \mathrm{C}_{\mathrm{i}}(\mathrm{t}) \mathrm{dt}
$$

Further coefficients are introduced to evaluate aerodynamic efficiency, power consumption and quality factor:

$$
\begin{gathered}
\overline{\mathrm{C}_{\text {eff }}}=\frac{\overline{\mathrm{C}_{\mathrm{y}}}}{\overline{\overline{\mathrm{C}_{\mathrm{d}}}}} \\
\overline{\mathrm{C}_{\mathrm{p}}}=\frac{1}{\mathrm{~T}_{0}^{\mathrm{T}}} \mathrm{C}_{\mathrm{d}}(\mathrm{t}) \frac{\mathrm{V}(\mathrm{t})^{3}}{\mathrm{~V}_{0}^{3}} \mathrm{dt} \\
\overline{\mathrm{C}_{\mathrm{q}}}=\frac{\overline{\mathrm{C}_{\mathrm{y}}} \frac{3}{2}}{2 \overline{\mathrm{C}_{\mathrm{p}}}} \sqrt{\frac{\mathrm{c}}{\mathrm{a}}}
\end{gathered}
$$

where $V(t)$ is the instantaneous airfoil velocity. The efficiency ratio represents the ratio between the effective lifting force necessary for flight and the drag force countering the wing motion, acting as a source of energy consumption. The power ratio may be assimilated to the power consumption resulting from the presence of this drag force. Finally, the Froude theory leads to the definition of a quality coefficient which estimates the relative importance of the lifting force and the power consumption. Note that in this case "a" stands for the total flapping amplitude.

\section{Results and discussion}

\subsection{Mean analysis}

The aerodynamic performances inherent to the different kinematics are evaluated using the mean coefficients described in the previous section. Figure 4 represents the mean drag, vertical (or lifting) force, efficiency ratio, power and quality coefficients for all the 
configurations tested. The latter is regrouped according to the value of the downstroke angle of attack $\alpha_{d}$ consecutively fixed to $30^{\circ}, 45^{\circ}$ and $60^{\circ}$. In this way, each graph exhibits the effect of desymmetrization on the global aerodynamic performances of flapping airfoils. Precisely, the right hand symbols correspond to symmetric cases, the asymmetry being increased with decreasing upstroke angle of attack.
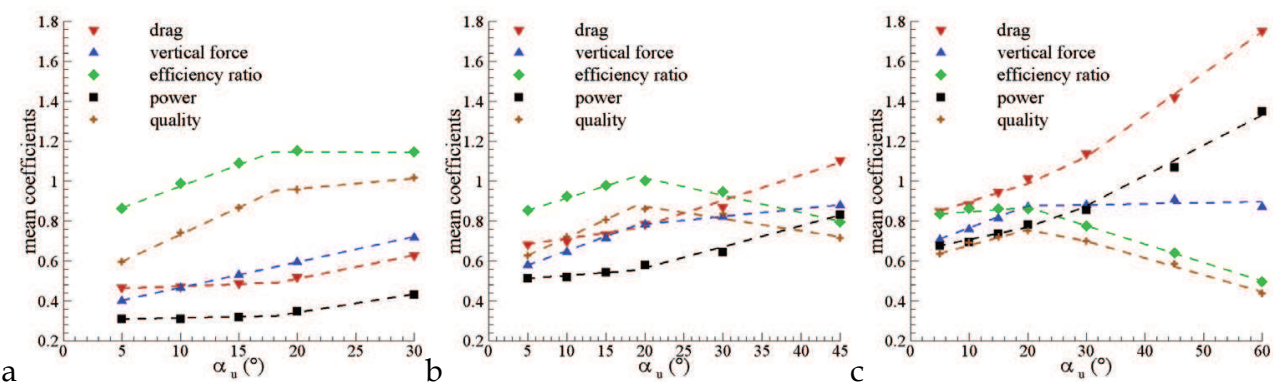

Figure 4. Mean drag, vertical force, efficiency ratio, power and quality $\left(5^{*} \overline{\mathrm{C}_{\mathrm{q}}}\right)$ coefficients in function of $\alpha_{\mathrm{u}}$ for the $\alpha_{\mathrm{d}}=30^{\circ}(\mathrm{a}), \alpha_{\mathrm{d}}=45^{\circ}(\mathrm{b})$ and $\alpha_{\mathrm{d}}=60^{\circ}$ (c) configurations

The overall comparison demonstrates that the mean drag and vertical force coefficients, hence the global aerodynamic force $\mathrm{F}$, increase with the downstroke/upstroke angle of attack, as denoted in previous works (Sane \& Dickinson, 2001; Wu \& Sun, 2004; Kurtulus, 2005). This remark is illustrated by both the enhancement of the drag and vertical force tendency curves from one graph to another (influence of $\alpha_{d}$ ) and the latter's positive slope observed within a graph (influence of $\alpha_{\mathrm{u}}$ ).

In order to characterize the effect of asymmetry on aerodynamic performances, it is of interest to further analyse the behaviour of these tendency curves within a graph. Figure 4.a shows a linear increase of the vertical force coefficient with the upstroke angle of attack. This augmentation is similar for the drag coefficient when $\alpha_{\mathrm{u}}$ is set above $18^{\circ}$, but less pronounced for low $\alpha_{\mathrm{u}}$ values. The direct consequence resides in a clear change of the efficiency ratio tendency curve near $\alpha_{\mathrm{u}}=18^{\circ}$. Comparable changes in evolution are further put into evidence for both drag and vertical force coefficients in figure 4.b. In this particular case, the behaviours of the drag and the vertical force seem opposed, resulting in a maximum efficiency ratio of approximately 1.05 at $\alpha_{\mathrm{u}} \approx 19^{\circ}$. Besides these changes, Figure 4.c exhibits a quasi constant vertical force coefficient and a sharp increase in drag with increasing upstroke angle of attack. Thus, the efficiency ratio is maintained to approximately 0.85 until $\alpha_{\mathrm{u}} \approx 20^{\circ}$, after which it is strongly affected and decreases to 0.498 for the normal hovering case $\alpha_{\mathrm{d}}=60^{\circ}, \alpha_{\mathrm{u}}=60^{\circ}$. As a conclusion, the common characteristic of all groups $\left(\alpha_{d}=30^{\circ}, \alpha_{d}=45^{\circ}\right.$ and $\left.\alpha_{d}=60^{\circ}\right)$ resides in the presence of a critical upstroke angle of attack for which the tendency curve representing the efficiency ratio as a function of $\alpha_{u}$ changes behaviour and reaches a strong (maximum in some cases) value. If the cause for the existence of this critical angle of attack may arise from trivial trigonometric considerations, it is also probable that the occurence of non-linear unsteady flow structures strongly affect aerodynamic performances from one configuration to another, and may participate in the changes of behaviour previously exposed.

Furthermore, in all cases, the drag and power coefficients are characterized by comparable tendencies, still shifted by a value which increases with the upstroke angle of attack. The 
effect on the quality coefficient is such that the latter demonstrates analogous changes around $\alpha_{\mathrm{u}} \approx 20^{\circ}$.

\begin{tabular}{|l|l|l|l|l|l|l|l|}
\hline $\mathrm{a}_{\mathrm{d}}$ & $\mathrm{a}_{\mathrm{u}}$ & $\overline{\mathrm{C}_{\mathrm{d}}}$ & $\overline{\mathrm{C}_{\mathrm{y}}}$ & $\overline{\mathrm{C}_{\text {eff }}}$ & $\overline{\mathrm{C}_{\mathrm{p}}}$ & $\overline{\mathrm{C}_{\mathrm{q}}}$ & $\beta$ \\
\hline 30 & 5 & 0.4651 & 0.4018 & 0.864 & 0.310 & 0.119 & 34 \\
\hline 30 & 10 & 0.4698 & 0.4647 & 0.989 & 0.309 & 0.148 & 24 \\
\hline 30 & 15 & 0.4863 & 0.5300 & 1.090 & 0.321 & 0.173 & 17 \\
\hline 30 & 20 & 0.5185 & 0.5966 & 1.151 & 0.348 & 0.191 & 10 \\
\hline 30 & 30 & 0.6275 & 0.7190 & 1.146 & 0.433 & 0.203 & 0 \\
\hline 45 & 5 & 0.6807 & 0.5802 & 0.852 & 0.512 & 0.125 & 51 \\
\hline 45 & 10 & 0.6983 & 0.6444 & 0.923 & 0.520 & 0.144 & 41 \\
\hline 45 & 15 & 0.7314 & 0.7149 & 0.977 & 0.543 & 0.161 & 33 \\
\hline 45 & 20 & 0.7823 & 0.7826 & 1.000 & 0.580 & 0.172 & 25 \\
\hline 45 & 30 & 0.8685 & 0.8217 & 0.946 & 0.644 & 0.167 & 14 \\
\hline 45 & 45 & 1.1038 & 0.8801 & 0.797 & 0.832 & 0.143 & 0 \\
\hline 60 & 5 & 0.8477 & 0.7080 & 0.835 & 0.676 & 0.127 & 64 \\
\hline 60 & 10 & 0.8805 & 0.7598 & 0.863 & 0.695 & 0.138 & 54 \\
\hline 60 & 15 & 0.9435 & 0.8134 & 0.862 & 0.735 & 0.144 & 45 \\
\hline 60 & 20 & 1.0129 & 0.8706 & 0.860 & 0.782 & 0.150 & 38 \\
\hline 60 & 30 & 1.1367 & 0.8811 & 0.775 & 0.855 & 0.140 & 25 \\
\hline 60 & 45 & 1.4182 & 0.9087 & 0.641 & 1.070 & 0.117 & 14 \\
\hline 60 & 60 & 1.7509 & 0.8713 & 0.498 & 1.350 & 0.087 & 0 \\
\hline
\end{tabular}

Table 2. Aerodynamic coefficients survey

\subsection{Flow unsteadiness}

Focusing on the spatial and temporal evolution of the vortical structures governing the flow dynamics appears as an essential step in the comprehension of the asymmetric flapping flight aerodynamic performances. In order to explain the different tendencies in the generation of lifting force and in the behaviour of the efficiency ratio from one configuration to another, the adimensional $\lambda_{2}{ }^{*}$ (figure 6) and pressure $C_{p}$ (figure 7) contours are displayed for the symmetric configuration $\alpha_{\mathrm{d}}=45^{\circ}, \alpha_{\mathrm{u}}=45^{\circ}$ and the asymmetric configuration $\alpha_{\mathrm{d}}=45^{\circ}$, $\alpha_{\mathrm{u}}=20^{\circ}$. These flow properties are correlated with the time-dependent lift and drag coefficients (figure 5), putting into evidence the force generating mechanisms. The two configurations show comparable downstroke kinematics, hence comparable quasi-steady aerodynamic forces, but different upstroke kinematics. As a consequence, differences observed in the flow dynamics and in the generation of forces during the downstroke phase directly arise from the previous strokes history, especially through the wake capture 
phenomenon. Note that the downstroke kinematics of both cases cannot be qualified as identical since the rotation velocities are not strictly equal.
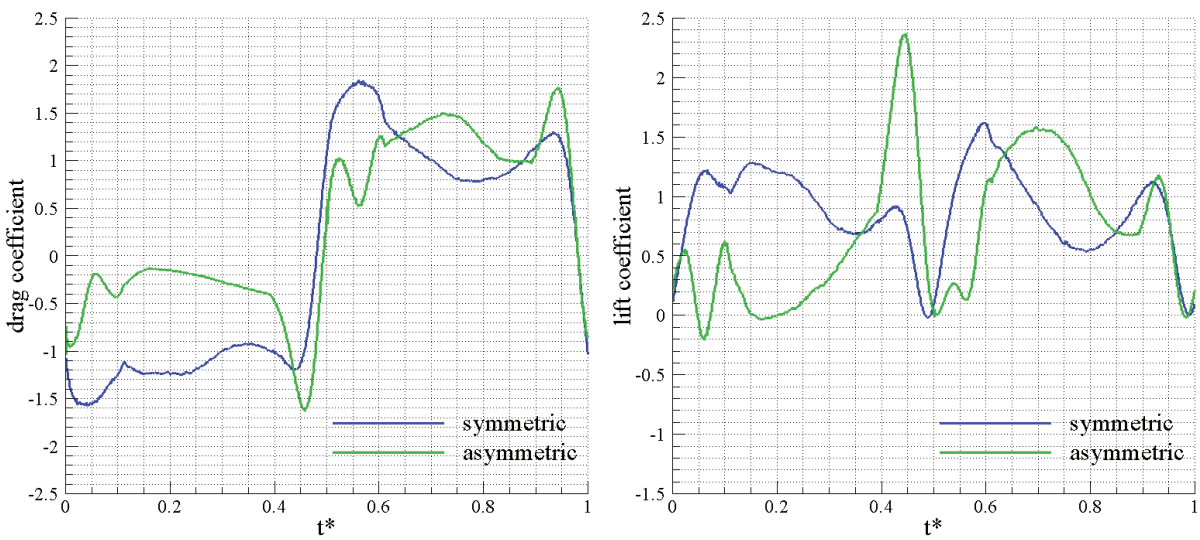

Figure 5. Drag and lift coefficient histories of the symmetric $\alpha_{d}=45^{\circ}, \alpha_{u}=45^{\circ}$ and asymmetric $\alpha_{\mathrm{d}}=45^{\circ}, \alpha_{\mathrm{u}}=20^{\circ}$ configurations

All flow properties and aerodynamic coefficients are represented at time $t^{*}$, where $t^{*}$ corresponds to the time elapsed since the beginning of the flapping cycle, adimensionalized by the period $\mathrm{T}$.

At $t^{*}=0$, the airfoil begins the upstroke phase. Notwithstanding the zero translating velocity which characterizes the flapping kinematics at this instant, the drag coefficients reach a strong value $(\approx-1)$ for both configurations. Indeed, two counter-rotating vortical structures dominate the flow in the vicinity of the profile (figure $6-t^{*}=0$ ), inducing the presence of a fluid jet directed towards the intrados and which acts as a drag enhancer. As previously expressed, this interaction between the airfoil and its own wake is typical of the flapping wings aerodynamics.

Between $t^{*}=0$ and $t^{*}=0.1$, the airfoil accelerates while still rotating to reach a constant translating speed and fixed angle of attack. The production of drag and lift here derives from the complex combination of inertial effects and vortex behaviours. Hence, significant differences can be observed between both symmetric and asymmetric configurations. On the one hand, the aerodynamic force resulting from the symmetric motion seems to primarily arise from the accelerating inertial effect, leading to the presence of a strong pressure region covering most of the airfoil intrados. On the other hand, the relative position of the airfoil and the previously shed vortices characterizing the asymmetric configuration induces a competition between strong (leading edge) and low (trailing edge) intrados pressure regions (figure $7-t^{*}=T / 12$ ). Therefore, at $t^{*}=0.06$, the asymmetric configuration lift and drag decrease sharply whereas the symmetric ones are maintained to relatively strong values.

At $t^{*}=0.1$, both cases demonstrate the formation of a LEV acting as a low pressure suction region on the extrados of the airfoil. Note that the latter is promoted by the wing/wake interaction as shown in previous studies (Birch \& Dickinson, 2003). The LEV is essential when considering the symmetric motion since it dominates the vortical flow field during most of the upstroke phase. Thus, absolute values of lift and drag above 1 are reached. On the contrary, the LEV generated in the asymmetric case has a limited lifetime such that its 
influence is only perceptible through the occurrence of a narrow lift peak. Note that this vortical structure does not significantly affect the drag since the upstroke angle of attack is set to a low value $\left(20^{\circ}\right)$, i.e. the airfoil surface projection on the axis perpendicular to the stroke plane is weak. Moreover, one can remark that the lifetime is partially shortened by the wake capture phenomenon which leads to the LEV shedding (figure $6-t^{*}=T / 12$ ). Consequently, the asymmetric upstroke phase leads to a partially attached flow supported by the presence of a fluid downwash engendered by the previous strokes.

The time evolutions of the drag and lift coefficients roughly respond to the spatial and temporal evolution of the upstroke LEV until $t^{*}=0.4$. At this instant, the airfoil starts to rotate while decelerating. Figure 5 shows the presence of drag and lift peaks, particularly pronounced in the asymmetric configuration for which the angle of attack changes from $\alpha_{\mathrm{u}}=20^{\circ}$ to $\alpha_{\mathrm{d}}=45^{\circ}$ with a rotating speed superior to the one encountered in the symmetric configuration. Such lift enhancements arise from the supplementary circulation generated by the combined rotating and translating motions (Kramer effect). Furthermore, whereas the rotation strengthens the Trailing Edge Vortex (TEV) formed during the latter part of the downstroke phase in the symmetric case, it is the cause for the formation of a Rotating Trailing Edge Vortex (RTEV) in the asymmetric case (figure $6-t^{*}=5 T / 12$ ).

At $t^{*}=0.5$, as observed at $t^{*}=0$, the drag coefficient resulting from the symmetric motion exhibits a strong value linked to the presence of a fluid jet generated by the two shed counter rotating vortical structures. The flow behaviour induced by the asymmetric motion considerably differs since no strong separated flow yielded to the presence of a vortex dipole (figure $6-t^{*}=6 \mathrm{~T} / 12$ ). Hence, any fluid jet tends to increase the drag coefficient which is here quasi null.

Between $t^{*}=0.5$ and $t^{*}=0.6$, the airfoil accelerates in the opposite direction to initiate the downstroke phase. Despite the symmetric aspect of the normal hovering case, the presence of highly unsteady non-linear structures leads to non-strictly equivalent upstroke and downstroke aerodynamic forces, as noticed by other authors on biologic cases (Van Oudheusden et al, 2008). However, global variations of upstroke and downstroke coefficients strongly resemble as arising from comparable flow behaviour. The symmetric motion engenders a rapid augmentation of lift and drag through the accelerating inertial effects. The latter is supported and maintained by the formation of a downstroke LEV on the airfoil extrados (figure $6-t^{*}=7 \mathrm{~T} / 12$ ). On the contrary, the influence of wake capture is reduced in the asymmetric case. Indeed, besides the action of the RTEV assimilated to a low pressure region under the intrados of the airfoil, the previously shed vorticity is not sufficient to accelerate the formation of a downstroke LEV. Consequently, the drag and lift are maintained to low values until $t^{*}=0.6$. The interesting aspect here resides in the fact that the LEV develops smoothly, staying closely attached to the extrados. Thus, from $t^{*}=0.6$, despite the fact that the wing kinematics are identical in both symmetric and asymmetric configurations (i.e. identical quasi-steady forces), the asymmetric motion leads to stronger aerodynamic coefficients. This observation is evidence that the wake capture phenomenon not only affects the early stages but most of the stroke resulting forces.

From $t^{*}=0.9$, the wake of both configurations still exhibit different behaviour hence different drag productions as the airfoil jointly rotates and decelerates. However, the lift coefficients tend to similar time evolutions since the inertial forces conducting the Kramer effect are quasi equivalent in both configurations. 


\subsection{Effects of desymetrization}

The correlation between the fluid dynamics and the loads experienced by the flapping airfoil for two specific symmetric and asymmetric configurations (respectively $\alpha_{\mathrm{d}}=45^{\circ}$, $\alpha_{\mathrm{u}}=45^{\circ}$ and $\alpha_{\mathrm{d}}=45^{\circ}, \alpha_{\mathrm{u}}=20^{\circ}$ ) demonstrated that reducing the upstroke wake by fixing a low upstroke angle of attack leads 1 ) to a reduction of the aerodynamic coefficients immediately after stroke reversal and 2) to an enhancement of the latter during most of the downstroke translating phase. This characteristic partially results from the absence of significant upstroke leading edge separation, hence significant wake capture, inducing the generation of a closely attached LEV on the extrados of the airfoil. The analysis of the unsteady drag and lift coefficients obtained for all configurations tested support this observation (figure 8). Note that each graph of figure 8 regroups the flapping kinematics parameterized with a common downstroke angle of attack such that it effectively illustrates the effect of desymetrization on the production of lift and drag during the downstroke phase (the quasi steady downstroke forces being equivalent). Nevertheless, analysing the lift and drag coefficients averaged over the downstroke phase shows that both effects seem to inhibit each other. For instance, the mean drag and lift coefficients obtained during the downstroke phase of the $\alpha_{d}=45^{\circ}, \alpha_{\mathrm{u}}=45^{\circ}$ and the $\alpha_{\mathrm{d}}=45^{\circ}, \alpha_{\mathrm{u}}=20^{\circ}$ configurations are respectively $1.09,0.87$ and $1.11,0.89$.

Besides the changes observed in the fluid dynamics, asymmetric hovering motions benefit from the combined action of both lift and drag as the effective lifting force (Wang, 2004). Indeed, for such motions, the hovering condition imposes the stroke plane to be inclined such that the drag has a vertical component and thus provides a lifting force rather than exclusively acting as a power consumer. According to the $\beta$ angles calculated and listed in table 1, the resulting horizontal and vertical unsteady components are deduced from the drag and lift components and represented in figure 9. In all cases, increasing the difference $\alpha_{d}-\alpha_{u}$, i.e. the stroke plane angle, considerably enhance the resulting downstroke vertical force. For instance, the mean lifting force coefficient obtained during the downstroke phase of the $\alpha_{\mathrm{d}}=45^{\circ}, \alpha_{\mathrm{u}}=45^{\circ}$ and the $\alpha_{\mathrm{d}}=45^{\circ}, \alpha_{\mathrm{u}}=20^{\circ}$ configurations are respectively 0.87 and 1.28 which corresponds to an increase of approximately $47 \%$. However, the upstroke phase appears as a harmful phase and may sometimes lead to a negative vertical force component as exhibited when the upstroke angle of attack is set to $10^{\circ}$. Note that such negative values can be obtained with still non-negligible angle of attack since the presence of a fluid downwash tends to reduce the effective angle of attack. Consequently, as dedicated to MAVs application, asymmetric kinematics with an upstroke angle of attack fixed below $15^{\circ}$ should be avoided. Moreover, the quality of the resulting aerodynamic performances resides in the relative importance of the upstroke and downstroke phases.

\subsection{Improving aerodynamic performances}

Previous sections showed that comparatively to symmetric flapping motions, asymmetric flapping motions may lead to sustained efficiency ratio coupled with a consistent lifting force when the upstroke angle of attack is lowered, weakening the wake capture phenomenon and implying the contribution of drag to the vertical force component. Furthermore, it obviously appeared that, the upstroke phase being harmful, the relative importance of the upstroke and downstroke phases should be deeply considered. In this brief section, we suggest a way to ameliorate the aerodynamic performances of asymmetric motions by increasing the upstroke translating velocity, i.e. reducing the relative importance 
of the upstroke phase. This approach is consistent since the inherent characteristics of the previous cases are not altered, the flow dynamics not depending on the Reynolds number in this range (Wu \& Sun, 2004; Kurtulus, 2005).

Results are obtained for velocity ratios (defined as the ratio $r_{v}$ between the upstroke and the downstroke translating velocity) of $1,1.2$ and 1.4. The aerodynamic coefficients are determined by adimensionalizing the upstroke and downstroke forces by means of the upstroke and the downstroke translating velocities respectively. Tables 3 and 4 illustrate the effect of increasing the upstroke velocity on two chosen asymmetric cases. It is shown that the lifting force and the efficiency can be increased in a prompter way than the power consumption such that the quality coefficient can be significantly enhanced. Indeed, one might observe that the influence on the power consumption is not monotonous (increasing between $r_{v}=1$ and $r_{v}=1.2$, decreasing between $r_{v}=1.2$ and $r_{v}=1.4$ ).

\begin{tabular}{|l|l|l|l|}
\hline & $\mathrm{r}_{\mathrm{V}}=1$ & $\mathrm{r}_{\mathrm{V}}=1.2$ & $\mathrm{r}_{\mathrm{V}}=1.4$ \\
\hline$\overline{\mathrm{C}_{\mathrm{d}}}$ & 0.7314 & $0.7497(+2.5 \%)$ & $0.7733(+5.7 \%)$ \\
\hline$\overline{\mathrm{C}_{\mathrm{y}}}$ & 0.7149 & $0.7825(+9.5 \%)$ & $0.8308(+16.2 \%)$ \\
\hline$\overline{\mathrm{C}_{\mathrm{eff}}}$ & 0.977 & $1.044 \quad(+6.9 \%)$ & $1.074 \quad(+9.9 \%)$ \\
\hline$\overline{\mathrm{C}_{\mathrm{p}}}$ & 0.543 & $0.604 \quad(+11.2 \%)$ & $0.583 \quad(+7.4 \%)$ \\
\hline$\overline{\mathrm{C}_{\mathrm{q}}}$ & 0.161 & $0.165 \quad(+2.5 \%)$ & $0.187 \quad(+16.1 \%)$ \\
\hline
\end{tabular}

Table 3. Effect of velocity ratio on aerodynamic coefficients for the asymmetric configuration $\alpha_{\mathrm{d}}=45^{\circ}, \alpha_{\mathrm{u}}=15^{\circ}$

\begin{tabular}{|l|l|l|l|}
\hline & $\mathrm{r}_{\mathrm{V}}=1$ & $\mathrm{r}_{\mathrm{V}}=1.2$ & $\mathrm{r}_{\mathrm{V}}=1.4$ \\
\hline$\overline{\mathrm{C}_{\mathrm{d}}}$ & 0.7823 & $0.7848(+0.3 \%)$ & $0.8144 \quad(+4.1 \%)$ \\
\hline$\overline{\mathrm{C}_{\mathrm{y}}}$ & 0.7826 & $0.8212(+4.9 \%)$ & $0.8498 \quad(+8.6 \%)$ \\
\hline$\overline{\mathrm{C}_{\mathrm{eff}}}$ & 1.000 & $1.046 \quad(+4.6 \%)$ & $1.043 \quad(+4.3 \%)$ \\
\hline$\overline{\mathrm{C}_{\mathrm{p}}}$ & 0.580 & $0.628 \quad(+8.3 \%)$ & $0.599 \quad(+3.3 \%)$ \\
\hline$\overline{\mathrm{C}_{\mathrm{q}}}$ & 0.172 & $0.171 \quad(-0.6 \%)$ & $0.189 \quad(+9.9 \%)$ \\
\hline
\end{tabular}

Table 4. Effect of velocity ratio on aerodynamic coefficients for the asymmetric configuration $\alpha_{\mathrm{d}}=45^{\circ}, \alpha_{\mathrm{u}}=20^{\circ}$ 


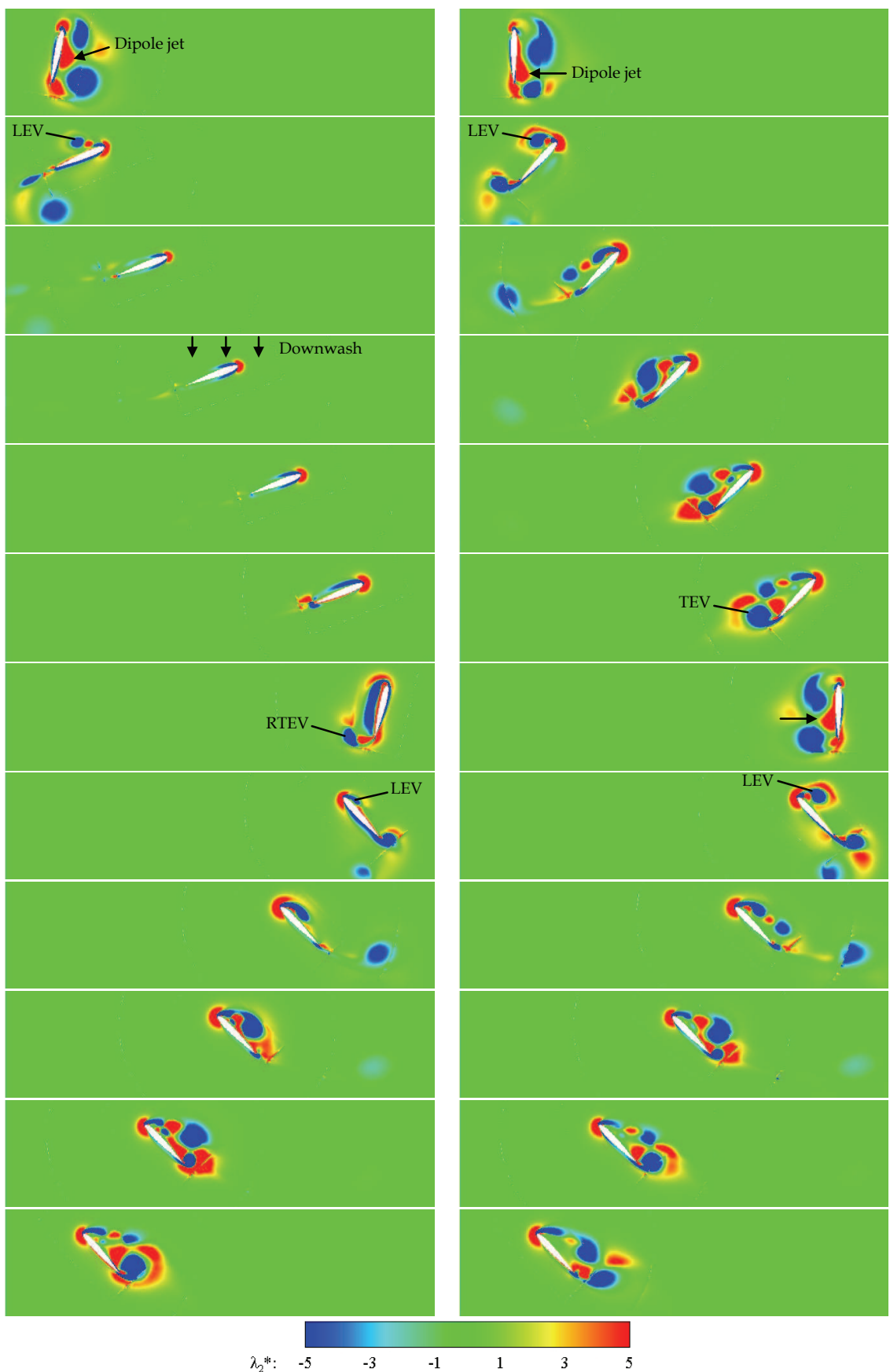

Figure 6. $\lambda_{2}{ }^{*}$ distribution at $t^{*}=n T / 12(n \in[0 ; 11])$ from top to bottom for the symmetric (right) and asymmetric (left) configurations 


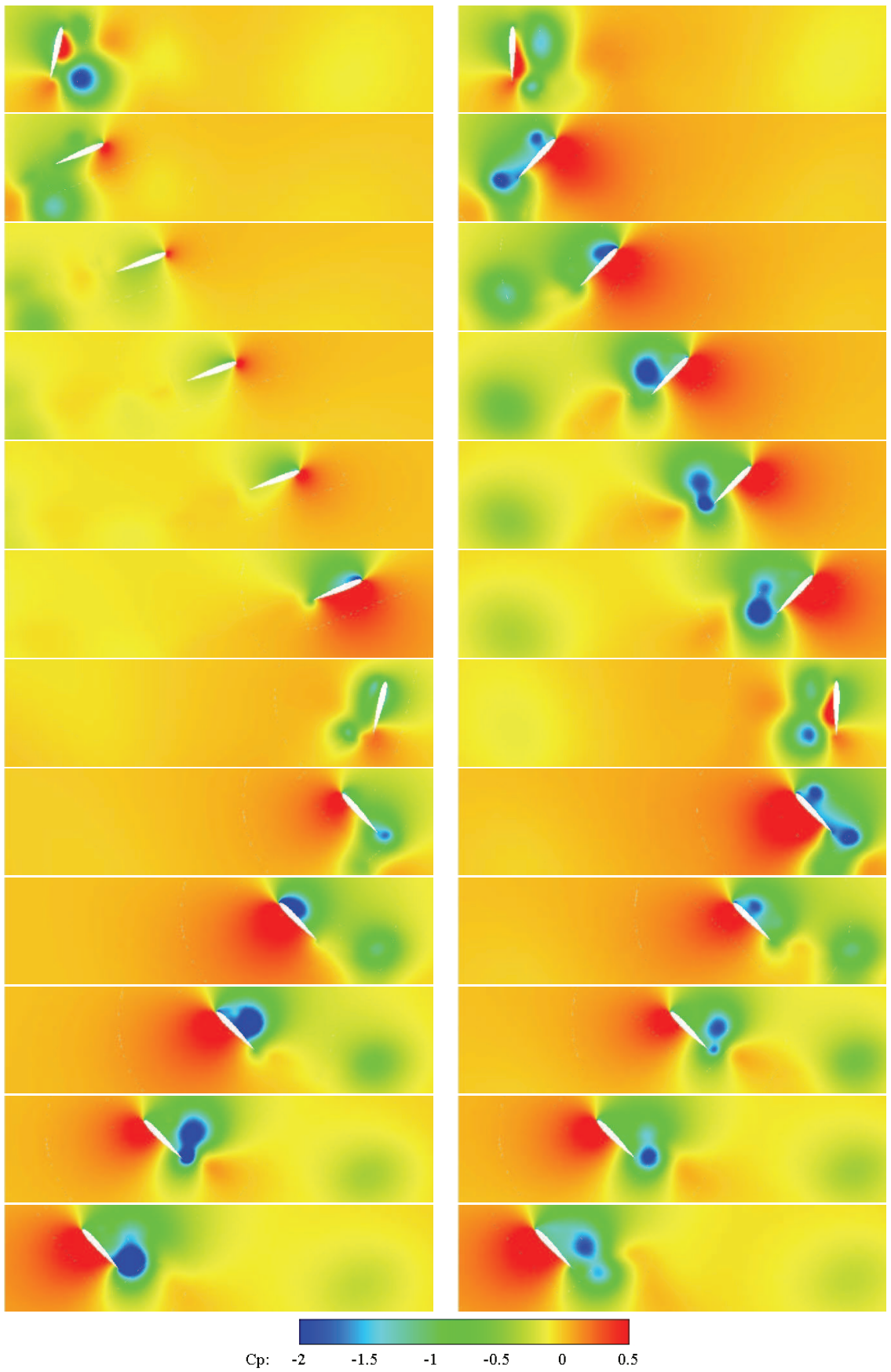

Figure 7. Adimensional pressure distribution at $\mathrm{t}^{*}=\mathrm{nT} / 12$ (n $\left.€[0 ; 11]\right)$ from top to bottom for the symmetric (right) and asymmetric (left) configurations 

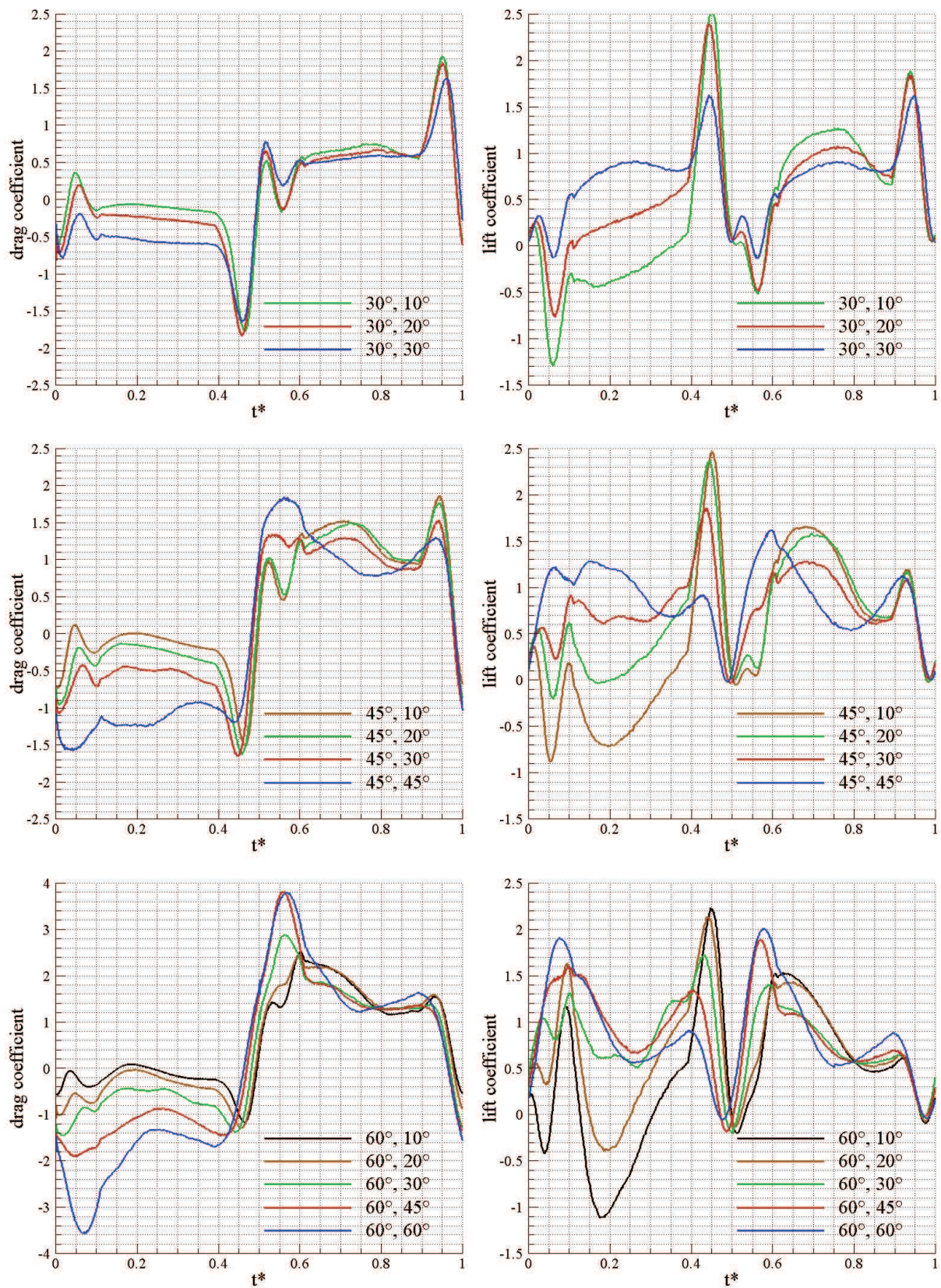

Figure 8. Drag and lift coefficient histories (configurations referenced with $\alpha_{\mathrm{d}}, \alpha_{\mathrm{u}}$ ) 

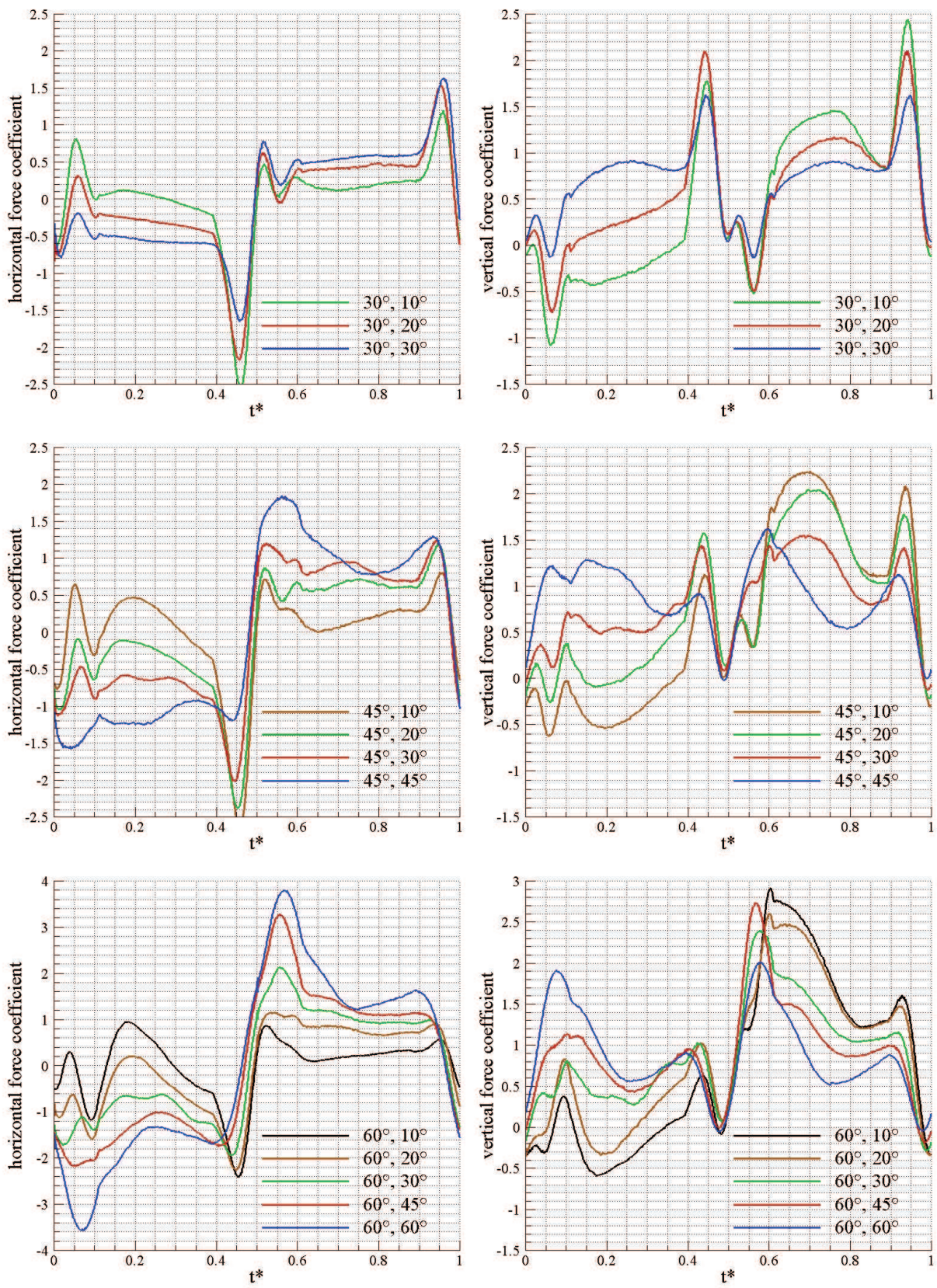

Figure 9. Horizontal and vertical force coefficient histories (configurations referenced with $\left.\alpha_{\mathrm{d}}, \alpha_{\mathrm{u}}\right)$ 


\section{Conclusion}

Micro Air Vehicles are characterized by limited dimensions $(15 \mathrm{~cm})$ which places the corresponding aerodynamic flow in the range of low Reynolds number flows $\left(10^{2}-10^{4}\right)$. At such Reynolds numbers, the flapping wing concept appears as an alternative solution to the conventional fixed and rotary wings, presupposing enhanced aerodynamic performances. Previous works relied on the analysis of normal (symmetric) hovering configurations as being the most common motion kinematics observed in the world of insects. In this study, parameterized asymmetric flapping motions at Reynolds 1000 are investigated by means of two-dimensional DNS calculations and compared to symmetric flapping motions.

In a first step, the mean analysis of the resulting aerodynamic coefficients demonstrate that introducing asymmetry (by differentiating the upstroke angle of attack to the downstroke one) generally lowers the aerodynamic force experienced by the airfoil while enhancing both efficiency and quality coefficients. Furthermore, when the latter is displayed as a function of the upstroke angle of attack, two distinct tendency behaviours are denoted on both sides of $\alpha_{\mathrm{u}} \approx 20^{\circ}$.

In a second step, the flow unsteadiness is analysed by means of the $\lambda_{2}$ and the pressure contours. The symmetric case parameterized with $\alpha_{d}=\alpha_{u}=45^{\circ}$ exhibits a strong leading edge separation during the upstroke translating phase, implying the occurrence of significant wing/wake interactions at stroke reversal. On the contrary, the asymmetric case is parameterized with $\alpha_{\mathrm{d}}=45^{\circ}, \alpha_{\mathrm{u}}=20^{\circ}$ such that the upstroke flow is attached, resulting in a reduced wake capture phenomenon. The direct consequences are 1) the absence of lift peak at stroke reversal and 2) the presence of a closely attached Leading Edge Vortex inducing enhanced downstroke lift. Such observations suggest that the behaviours observed on mean coefficients might arise from the presence or not of strong separation during upstroke. Moreover, the hovering condition applied on asymmetric motions imposes the stroke plane to be inclined such that part of the drag provides a lifting force rather than exclusively consuming power. Hence, the benefit of a closely attached LEV producing both lift and drag is further increased.

Despite the generation of enhanced downstroke lifting force, asymmetric configurations are characterized by a harmful upstroke phase. This aspect partially engenders a decrease in mean resulting aerodynamic forces. However, in order to improve global performances, it is of interest to shorten the latter, weakening the relative importance of upstroke comparatively to downstroke.

Consequently, this chapter brings further insight into the aerodynamics of asymmetric flapping motions and provides interesting perspectives for the development of high efficiency/quality Micro Air Vehicles.

\section{References}

Bennett, L. (1970). Insect flight: lift and the rate of change of incidence. Science, 167, 177-179 Birch, J.M., Dickinson, M.H. (2003). The influence of wing-wake interactions on the production of aerodynamic forces in flapping flight. Journal of Experimental Biology, 206, 2257-2272

Bos, F.M., Lentink, D., Van Oudheusden, B.W., Bijl, H. (2008). Influence of wing kinematics on aerodynamic performance in hovering insect flight. Journal of Fluid Mechanics, $594,341-368$ 
Dickinson, M.H., Götz, K.G. (1993). Unsteady aerodynamic performance of model wings at low Reynolds numbers. Journal of Experimental Biology, 174, 45-64

Dickinson, M.H., Lehmann, F.O., Sane, S.P. (1999). Wing rotation and the aerodynamic basis of insect flight. Science, 284, 1954-1960

Ellington, C.P. (1984). The aerodynamics of hovering insect flight I-V. Philosophical Transactions of the Royal Society. Series B: Biological Sciences, 305, 1122, 1-181

Jensen, M. (1956). Biology and physics of locust flight. III. The aerodynamics of locust flight. Philosophical Transactions of the Royal Society. Series B: Biological Sciences, 239, 667, 511-552

Jeong, J., Hussain, F. (1995). On the identification of a vortex. Journal of Fluid Mechanics, 285, 69-94

Kramer, M. (1932). Die zunahme des maximalauftriebes von tragflugeln bei plotzlicher anstellwinkelvergrosserung. Z Flugtech Motorluftschiff, 23, 185-189

Kurtulus, D.F. (2005). Numerical and experimental analysis of flapping motion in hover. Application to Micro Air-Vehicles. Phd Thesis, University of Poitiers, Laboratoire d'Etudes Aérodynamiques

Maxworthy, T. (1979). Experiments on the Weis-Fogh mechanism of lift generation by insects in hovering flight Part 1. Dynamics of the 'fling'. Journal of Fluid Mechanics, 93, 47-63

Polhamus, E.C. (1971). Predictions of vortex-lift characteristics by a leading-edge suction analogy. Journal of Aircraft, 8, 4, 193 -199

Sane, S.P., Dickinson, M.H. (2001). The control of flight force by a flapping wing: lift and drag production. Journal of Experimental Biology, 204, 2607-2626

Sane, S.P. , Dickinson, M.H. (2002). The aerodynamic effects of wing rotation and a revised quasi-steady model of flapping flight. Journal of Experimental Biology, 205, 1087-1096

Walker, P.B. (1931). Growth of circulation about a wing and an apparatus for measuring fluid motion. ARC report

Wang, Z.J. (2004). The role of drag in insect hovering. Journal of Experimental Biology, 207, $4147-4155$

Weis-Fogh, T. (1973). Quick estimates of flight fitness in hovering animals, including novel mechanism for lift production. Journal of Experimental Biology, 59, 169-230

Wu, J., Sun, M. (2004). Unsteady aerodynamic forces of a flapping wing. Journal of Experimental Biology, 207, 1137-1150 


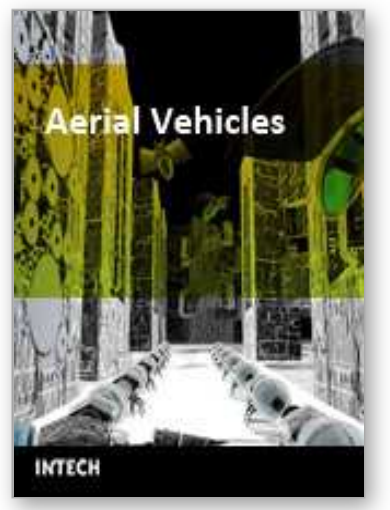

\author{
Aerial Vehicles \\ Edited by Thanh Mung Lam
}

ISBN 978-953-7619-41-1

Hard cover, 320 pages

Publisher InTech

Published online 01, January, 2009

Published in print edition January, 2009

This book contains 35 chapters written by experts in developing techniques for making aerial vehicles more intelligent, more reliable, more flexible in use, and safer in operation. It will also serve as an inspiration for further improvement of the design and application of aeral vehicles. The advanced techniques and research described here may also be applicable to other high-tech areas such as robotics, avionics, vetronics, and space.

\title{
How to reference
}

In order to correctly reference this scholarly work, feel free to copy and paste the following:

Jardin Thierry, Farcy Alain and David Laurent (2009). Asymmetric Hovering Flapping Flight: a Computational Study, Aerial Vehicles, Thanh Mung Lam (Ed.), ISBN: 978-953-7619-41-1, InTech, Available from: http://www.intechopen.com/books/aerial_vehicles/asymmetric_hovering_flapping_flight_a_computational_stu dy

\section{INTECH}

open science | open minds

\section{InTech Europe}

University Campus STeP Ri

Slavka Krautzeka 83/A

51000 Rijeka, Croatia

Phone: +385 (51) 770447

Fax: +385 (51) 686166

www.intechopen.com

\section{InTech China}

Unit 405, Office Block, Hotel Equatorial Shanghai

No.65, Yan An Road (West), Shanghai, 200040, China 中国上海市延安西路65号上海国际贵都大饭店办公楼405单元

Phone: +86-21-62489820

Fax: +86-21-62489821 
(C) 2009 The Author(s). Licensee IntechOpen. This chapter is distributed under the terms of the Creative Commons Attribution-NonCommercialShareAlike-3.0 License, which permits use, distribution and reproduction for non-commercial purposes, provided the original is properly cited and derivative works building on this content are distributed under the same license. 